\title{
Results of studies on extending the time operation of gin and linter grates
}

\author{
Salokhiddin Yunusov' ${ }^{1}$, Azamat Sultonov², Mashkhur Rakhmatov², Tojiddin Bobomurotov ${ }^{3}$, \\ and Mirkhosil Agzamov ${ }^{3 *}$ \\ ${ }^{1}$ Branch of Russian State University of Oil and Gas, 100125 Tashkent, Uzbekistan \\ 2"'Bukhara agroklaster" LLC, 200103, Bukhara, Uzbekistan \\ ${ }^{3}$ Tashkent Institute of Textile and Light Industry, 100100 Tashkent, Uzbekistan
}

\begin{abstract}
Results of studies on extending the time operation of gin and linter grates by hardening of the working area by high-frequency current are given in article. Mathematical models for calculation of key parameters of the mode of hardening of the working area of gin and linter grates are made. As a result of the executed researches the following conclusions are drawn: change of a gap between grates in a grate lattice leads to change of parameters of process of ginning and finally to deterioration of products. Mathematical models for calculations of parameters of process of hardening of the working area of grates are received. On the received mathematical models key parameters of hardening process current of high frequency which have the following values are calculated: depth of the tempered layer $-2.0 \mathrm{~mm}$, the optimum frequency of current -60000 , specific power $-500 \mathrm{~W} / \mathrm{cm}^{2}$, hardening time -10 , studying hardness tempered and not tempered sites of grates by method of Rockwell showed increase in hardness of the tempered site to HRC 55 against HRC 45 at not tempered by the working area.
\end{abstract}

\section{Introduction}

It is known that one of "mass" details of saw gin is the grate and in many respects key indicators of process of ginning depend on a condition of a grate. At the moment on many domestic saw gins the grates made of gray cast iron are used [1]. Technological process of production of pig-iron grates includes preparation of burdening materials, metal smelting, preparation of forming mixes, production of the top and lower semi-forms, loading, filling, unloading of a form, knockout casting from a form, its cleaning, stripping, check, machining [2].

Shortcomings of such grate, are apparently from listed above big labor input at production, the high prime cost, low wear resistance in the working area, short service life, lack of a possibility of adjustment at assembly in a lattice by bending because of fragility of cast iron, low maintainability, but also the steel grates made on the following method are used: produce preparation from round steel, then heat it to temperature of plastic

\footnotetext{
*Corresponding author: agzamov85@mail.ru
} 
deformation, form by rolling in three or four-roll calibers the required profile, cut on pieces of the required length which then bend on a profile, form the basic ends by forging, mechanically process a landing surface, make through hardening of the working area of a grate heating by means of the currents of high frequency installation, with the subsequent cooling in water [3-5].

Shortcomings of such grate are high fragility in the working area owing to through hardening, big labor input at production, the high cost, lack of a possibility of adjustment at assembly in a lattice by bending because of fragility, low maintainability (the need for preliminary "tempering" before surfacing up of a new layer of metal) [6-8].

Proceeding from the listed above shortcomings of the grates existing a way of production the task - maintaining flexibility on all length was set that at installation of a grate in a grate lattice gives more facilitated chance to carry out adjustment in the place by bending or straightening, improvement of maintainability, reduction of costs for recovery repair, reduction of time of hardening, reduction of an expense of the electric power, increase in labor productivity at production, decrease in cost of production.

\section{Solution to the Problem}

As a result of the solution of a task the technical result which consists in the following is received [9]:

1. The flexibility of a grate in the working area is kept because only the grate layer is tempered, and the core remained not tempered. It is known that usual, through hardening sharply increases fragility of a detail. In the proposed solution, due to application of hardening on the offered depth $(2 \mathrm{~mm})$ and the sizes $(40$ and $80 \mathrm{~mm})$, only the fragility of the small site increases and at the same time the plasticity of a core remains. Thanks to it the approximate, uniform plasticity on all length of a grate remains that facilitates installation of a grate in a grate lattice as with certain plasticity it is possible to carry out as required adjustment of a grate in the place by bending or straightening.

2. Improved maintainability, because during operation, the knee surface wears out and after complete wear of this layer, a non-knee, "raw" surface is exposed, on which surfacing with ordinary electrodes is easy.

3. Additional expenses at repair are excluded because such grate does not require before surfacing up carrying out such operations as a tempering and normalization which are required for surfacing up for grates with through hardening.

4. The hardening process duration since the small site is exposed to hardening is considerably reduced and its warming up requires several times less time in comparison with through warming up. Reduction of duration of process of hardening in turn leads to decrease in an expense of the electric power, increase in labor productivity at production of grates, to decrease in cost of production of grates.

Fig. 1 shows a side and front view of a grate, which consists of a lower leg (1), a grate body (2) and an upper leg (3).

The raw cotton fed into the working chamber of the saw gin is captured by the saw teeth of the saw cylinder and transported to the working area of the grate. In the working area, the fibers captured by the saw teeth pass into the gap formed between the grates, are separated from the seeds and carried further through the process, and the seeds roll down the bed of the grates and leave the working chamber [10].

An important factor in the operation of the saw-grate system is the wear of the lateral surface of the grate in the working area, leading to an increase in the number of defects in the cotton fiber. Studies have shown that after 6 months of gin operation, the gap between the grates increased to $5 \mathrm{~mm}$, with an allowable $3,8 \mathrm{~mm}$. With an increase in the gap, the number of defects in the cotton fiber increases - fragmentation, debris, the content of skin 
and broken seeds in the fiber. The main reason for the wear of the side surface of the grate is contact with the saw, which occurs due to inaccuracy in the assembly of the saw grate system [11].
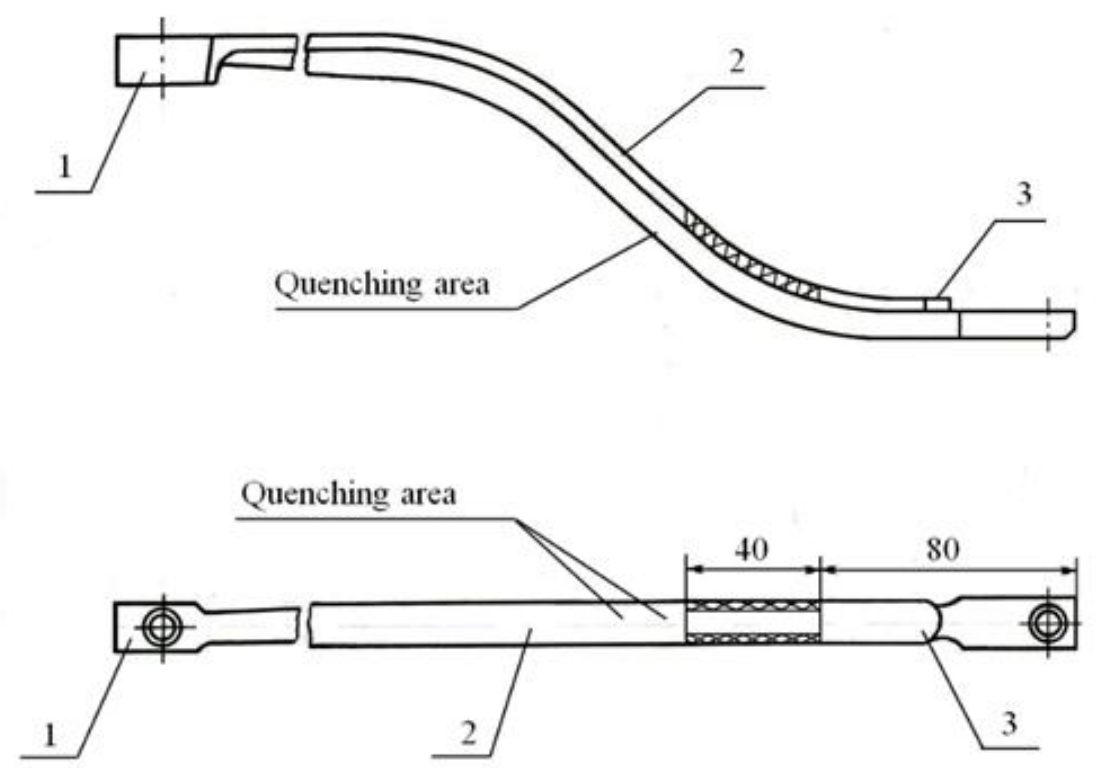

Fig. 1. Side and front view of a grate

The analysis of the design of the saw-grate system, from the point of view of the functional purpose of accuracy, manufacturability and reliability, carried out in order to determine the directions for improving the grate grates, made it possible to establish that with the existing technological process of manufacturing and assembling grates, it is not possible to ensure the required dimensional accuracy [12]. The assembly of grates in cotton factories is extremely laborious and requires a lot of highly skilled labor to perform fitting work, and, in addition, the design of the elements and the assembly technology used cannot provide the required accuracy of both the grate gaps themselves and their mutual arrangement [4]. The applied principle of assembling grate grates according to the method of individual fitting follows not because of technological necessity, but because of the unresolved issues of accuracy, as well as the manufacturability of the design of the grate itself and its elements.

Analysis of the accuracy of the mutual arrangement of the elements of the saw-grate system will make it possible to establish the presence of significant deviations of the system elements from the nominal dimensions, which are determined by the accuracy of the manufacture of grates, saws and gaskets, as well as the accuracy of their assembly. It was found that over $20-22 \%$ of the system elements work with interference, i.e. the grates of the saws.

In accordance with the technological regulations of work, the resource of the grate is 46 months, i.e. 2 sets of grates for 1 car are used per season. Despite the increased hardness (HRC 41) in the working area, the resistance of the grates is insufficient. In this regard, thousands of tons of cast iron are consumed annually for the manufacture of grates $[1,11]$.

Much attention was paid to the issues of increasing the service life of the grates. One of the most effective ways is the use of a wear-resistant replaceable element in the working area, which is practiced at cotton ginning plants in the United States. The use of a replaceable element makes it possible to reuse the grate itself by replacing the replaceable 
element at regular intervals, when the wear limit is reached. At cotton ginning plants, it is practiced to restore worn-out grates by surfacing followed by mechanical processing of the restored part.

Thus, for reliable operation of the saw grate system, it is necessary to ensure optimal roughness and sufficient wear resistance of the working surfaces of the grate. The material of the working surface of the grate can be selected only on the basis of experiments to determine the wear resistance of various materials under given operating conditions.

The most optimal way to increase the durability of steel grates is hardening, and to preserve the plastic properties of the grate, the most effective is surface hardening of the working area [9].

Currently, high-frequency heat treatment is used in the industry for surface hardening. High-frequency surface hardening is used in industry to obtain products with a hard wearresistant surface layer and a relatively soft and viscous core [12].

The first and main advantage of any surface heating hardening, including highfrequency surface hardening, in comparison with through hardening, is the reduction in the brittleness of parts and products. Brittleness decreases as a result of the formation of a plastic and viscous core, and in many cases due to the local location of the hardened layer only in areas whose hardness should be high.

Cracks and fractures during overloading and impacts, as well as in the presence of undercuts, holes, cross-sections and metal defects occur more easily in parts with through hardening than in parts with a surface hard layer. Cracks in through-hardened parts during production or operation lead to immediate or very rapid fracture. With surface hardening, cracks formed in the hard layer do not propagate into the soft and tough core.

Due to the significant reduction in the brittle fracture tendency achieved in the transition from through hardening to surface hardening, the tolerance limits for hardness and wear resistance are increased.

The second advantage of surface heating quenching is the significant reduction in deformation during heating and cooling, achieved by the stiffness of the cold core.

The third advantage is the almost complete elimination of oxidation and decarburization, which, while reducing deformation, makes it possible in some cases to quench the finished parts without grinding.

As you know, to obtain high wear resistance of cast iron gin grates, the working area is "bleached", in which the hardness of the working area surface is within HRC $56[4,9]$.

With surface hardening, used for the manufacture of grates, it is possible to obtain a hardness of HRC 58-62. However, fluctuations in the composition, hardening modes, as well as the need for self-tempering or tempering to combat cracks, lead to the fact that the lower permissible hardness limit in practice can be underestimated to HRC 54, which is approximately on the same level with the hardness of the bleached grate.

An important role for the high-quality hardening of the working surface of the grate is played by the correct choice of the main parameters of the inductor, heating rate, current frequency, heating time, cooling system, etc.

A feature of high-frequency induction heating of metals is the generation of heat energy directly in the heated product. Such heating determines the nature of the temperature change over the section of the heated grate.

With surface induction heating, holding is in most cases unacceptable or undesirable, since the effect of surface heating is lost due to thermal conductivity. The limited heating time may not be sufficient to complete the phase, structural transformations when heated to the temperatures recommended for the given steel. The completeness or degree of transformations is determined, in addition to the final heating temperature, by the residence time of the steel in the temperature range of phase transformations - the total austenitization time [8-10]. 
The temperature distribution over the cross section of a steel product during induction heating depends on the following factors: the depth of penetration of current into the metal, thermal conductivity of steel, heating rate, surface temperature, size and shape of the product.

In order to select the hardening parameters, we will consider some theoretical layouts.

To increase the stability period of the ginning process, it is necessary to increase the wear resistance of the grates. As mentioned above, one of the ways to achieve this goal is to harden the working surface. Below we will consider some theoretical aspects of the process of increasing the wear resistance of the grate and, consequently, the stability of the ginning process. Let us apply for calculations a well-known and relatively simple mathematical apparatus developed by a number of authors for solving problems of external heating [6-8]. The finiteness of the layer in which heat is released is taken into account by empirical corrections.

\section{Methodology}

When heat propagates in a homogeneous isotropic medium, it can be assumed that the direction of the heat flux coincides with the direction of the temperature gradient. We assume that the heat capacity $\mathrm{C}$ and the thermal conductivity $\sigma$ are constant, then the heat transfer equation can be written:

$$
\frac{\partial T}{\partial t}=\frac{\sigma}{\gamma C} \frac{\partial^{2} T}{\partial z^{2}}
$$

The coefficient facing the right part of this equation and representing the heat conductivity relation to volume thermal capacity is thermal diffusivity coefficient.

For different grades of cast iron its size fluctuates in quite wide limits: from 0.03 to 0.15 $\mathrm{cm}^{2} / \mathrm{sec}[9]$.

Let's designate further thermal diffusivity by the letter à. Therefore, the equation (1) can be rewritten:

$$
\frac{T}{\partial t}=\grave{a} \frac{\partial^{2} T}{\partial z^{2}}
$$

This equation completely coincides with the equations describing distribution of electromagnetic waves in metal.

When temperature on the surface of a body is periodic function from time, fluctuating around some average temperature of $T_{a v}$,

$$
F(t)=A \cos \omega t+T_{a v}
$$

The solution of the equation (2) will give the running fading wave similar to an electromagnetic wave in the metal conductor:

$$
T=T_{a v}+A_{\exp }\left(-z \sqrt{\frac{\omega}{2 a}}\right) \cos \left(\omega t-\sqrt{\frac{\omega}{2 a}} z\right)
$$

By analogy with an electromagnetic wave size $\sqrt{2 a / \omega}$ it is possible to call depth of penetration of a thermal wave. Let's designate further it by a letter $\rho$. At distribution on distance $\rho$ amplitude of a thermal wave changes in e of times. For points where $z \gg \rho$, temperature remains to invariable and equal $T_{a v}$. 
If to express duration of the period of a thermal wave $\tau$ in seconds, then for different grades of steel depth of its penetration will be:

$$
\rho_{F_{e}}=(0,1-0,2) \sqrt{\tau}
$$

The solution of the equation (2) taking into account that, on the heated surface constant temperature will be maintained, and the constant heat flux can be written down in the form of Crump's function. At the same time we will take for reference temperature $0^{\circ} \mathrm{C}$ will designate temperature on the $T$ grate surfaces.

At $T_{s}=$ const, temperature for any point in metal is defined by expression:

$$
T=T_{s} \cdot\left\{1-\Phi\left(\frac{z}{2 \sqrt{a t}}\right)\right\}
$$

Where sign $\Phi$ designates Gauss's integral [10].

Since it is necessary to temper the grate from both sides, we assume that the grate is a plate that must be heated from both sides and at the same time heat transfer from the end surfaces occurs.

When the plate thickness - $\mathrm{u}$ is exposed to heating, and $T_{s}$ temperature on both sides of a plate is maintained by a constant, temperature in the thickness of metal is defined by the equation

$$
T=T_{S} \cdot\left\{1-\frac{4}{\pi} \sum_{m=1}^{\infty} \exp \left[-\frac{2 m+1 \pi^{2} a}{u^{2}} t\right] \times \frac{\sin (2 m+1) \frac{\pi}{u} z}{2 m+1}\right\}
$$

From the equation (6) it is possible to define temperature at the top of the grate surface:

$$
\frac{\partial T}{\partial z}=\frac{T_{S}}{2 \sqrt{\pi a t}}
$$

Having multiplied this size, by heat conductivity of steel $\sigma$, we will receive the size of specific power which has to be attached to a grate surface for maintenance of constancy of temperature of a surface:

$$
\Delta P=\frac{\partial T}{\partial z} \sigma=\frac{\sigma T_{S}}{2 \sqrt{\pi a t}}
$$

For steel 45 it is possible to accept $\Delta P=0,55 \frac{T_{s}}{\sqrt{t}}, W / \mathrm{cm}^{2}$.

The full amount of the energy which passed through surface unit deep into of a grate will be equal:

$$
\Delta q=\int_{0}^{t} \Delta P d t=\frac{\sigma T_{S}}{\sqrt{\pi a}} \int_{0}^{t} \frac{d t}{2 \sqrt{t}}=\frac{\sigma T_{S}}{\sqrt{\pi f}} \sqrt{t}
$$

Following from the equation (9) that at the time of $t=0$ infinitely big powerto have temperature, other than zero, on the surface of steel. Thus the heating mode at constancy of external temperaturebe almost carried out, only since some time of $t$ which 1 the is less than more maximum specific power $\Delta P$, which we can have.

Let's pass to calculations of the mode of heating at the set size of the power allocated for surfaces. At $\Delta P=$ const the solution of the equation (2) has an appearance: 


$$
\left.T=\frac{2}{\sigma} \sqrt{a}\left\{\frac{1}{\sqrt{\pi}} \exp \left(-\frac{z}{\sqrt{a t}}\right)\right)-\frac{z}{2 \sqrt{a t}}\left[1-\Phi\left(\frac{z}{2 \sqrt{a t}}\right)\right]\right\} \Delta P \sqrt{t}
$$

Sign $\Phi$ as well as in the equation (6), designates Gauss's integral [10]. Specific power on a grate surface (at $z=0$ )

$$
T_{S}=\frac{2}{\sigma} \sqrt{\frac{a}{\pi}} \Delta P \sqrt{t}
$$

Considering that grate cast iron expression (12) can be rewritten:

$$
T_{S}=(0,5-1,5) \Delta P \sqrt{t}
$$

Let's accept for a grate as average value of the coefficient standing before $\Delta P$ a formula (4), size 0,7 and on the basis of the equation (7) we will construct two schedules of temperature change on time and on coordinate.

When heating under superficial hardening, external sources of heat, the heating mode usually corresponds, to constancy of specific power on the surface of hardening.

At induction heating the power brought to a grate can change in quite wide limits. A considerable part of time heating can go on the mode close to constancy of temperature on surfaces.

The same thickness of the tempered layer $\delta$ it is possible will receive at various specific capacities $\Delta P$ and durations of heating time t. Therefore on the basis of one size $\delta$ it is impossible to carry out still the choice of the mode of heating under hardening [11].

The transition layer between the martensitic zone and the core metal is denoted by $\beta$. The surface of temperatures will be internal border of this layer $A_{C_{1}}$. Heating is lower $A_{C_{1}}$ does not prepare steel for heat treatment. Temperature will be external border of a transitional layer $A_{C_{3}}$ when duration of heating is so big that the solid solution heated to $A_{C_{3}}$, it manages to be homogenized. At small times of heating the upper bound of a transitional layer should be considered a surface of temperatures, several highest, than $A_{C_{3}}$.

It is known that depending on composition of steel and speed of heating the difference of temperatures on borders of a transitional layer, can fluctuate from 50 to 2000C Pages. Further, we will designate temperature on borders of a transitional layer $T_{\beta_{1}}$ and $T_{\beta_{2}}$.

By the time of the end of process of heating under superficial hardening transitional the layer consists of the structures which did not completely lose the magnetic permeability. Therefore, even when heating by currents of low frequency, when $\delta<600 \sqrt{f}$, depth of penetration of current in a transitional layer turns out less than its thickness $\beta$.

Therefore it is accepted that in transitional heat passes a layer in the basic due to heat conductivity. At such assumption it is possible to determine that specific power which has to be attached to a grate at the time of the end of heating to receive transitional a layer of the required thickness,

$$
\Delta P=\frac{\sigma\left(T_{\beta_{1}}-\beta_{2}\right)}{\beta}
$$

If are set thickness of a transitional layer $\beta$ the difference of temperatures on its borders $T_{\beta_{1}}$ and $T_{\beta_{2}}$, it is possible to define coefficient of excess heat analytically: 


$$
\mathrm{K}=1+\frac{\mathrm{T}_{\beta 1} \beta}{2\left(\mathrm{~T}_{\beta 1}-\mathrm{T}_{\beta 2}\right) \delta}
$$

Size of overheating of an outer surface $\Delta T$ depends not only from $T_{\beta_{1}}-T_{\beta_{2}}$. It is possible to choose so the frequency of current and duration of heating that overheating and at a narrow transitional zone was very small.

If to accept that full amount of energy which needs to be reported to a grate for preparation it to hardening, equally $K \cdot 5000 \delta \mathrm{W} \cdot \mathrm{sek}$, on the basis of formulas (14) and (15) it is possible to calculate heating duration under hardening:

$$
t=\frac{\mathrm{K} \cdot 5000 \delta}{\Delta \mathrm{P}_{c p}}=\frac{\mathrm{K} \cdot 5000 \delta}{\sigma\left(\mathrm{T}_{\beta 1}-\mathrm{T}_{\beta 2}\right) \frac{\Delta \mathrm{P}_{c p}}{\Delta \mathrm{P}_{\text {кон }}}}
$$

This equation approximately can be written down:

$$
t \approx \frac{5000 \beta^{2} T_{1}}{2 \sigma\left(T_{\beta_{1}}-T_{\beta_{2}}\right)^{2}\left(\frac{\Delta P a v}{\Delta P_{c o n}}\right)} \approx 5 \cdot 10^{3} \frac{T_{\rho 1}}{\left(T_{\beta_{1}}-T_{\beta_{2}}\right)^{2}} \beta^{2}
$$

Thus, one may say, that heating duration under superficial hardening is proportional to a square of thickness of a transitional zone.

Taking into account that thickness of a transitional layer usually is $0.3-0.5$ from the tempered layer, on the basis of the above-stated theoretical calculations it is possible to calculate heating time duration in dependence of thickness of the tempered layer.

Let us find the temperature distribution over the grate surface in a stationary mode, i.e. when the current in the inductor is adjusted so that a constant temperature $(\mathrm{T})$ is maintained on the side surface of the grate. The heat transfer from the end surface of the grate is assumed to obey Newton's law, i.e. we will assume that the amount of heat given off is proportional to the temperature: $q=k T$. We will not take into account the temperature difference along the grate height [12].

Let's allocate in a grate the site at distance $x$ will make for it balance of energy. Arrival of heat from the periphery of a grate will be equal:

$$
q_{1}=2 \pi(x+\Delta x) h \sigma\left(\frac{d T}{D x}\right)_{x+\Delta x}
$$

Losses of energy on a heat transfer and radiation

$$
q_{2}=2 \pi x h \sigma\left(\frac{d T}{d x}\right)_{x}=2 \pi x \Delta x k T
$$

On the basis of these two ratios it is possible to work out the heat transfer equation:

$$
\frac{d^{2} T}{d x^{2}}+\frac{1}{x} \frac{d T}{d x}-\frac{k}{h \sigma} T=0
$$

Having designated size $x \sqrt{\frac{k}{h \sigma}}=u$ having entered this new variable into the equation 
(20), we will receive the usual equation of Bessel of a zero order:

$$
\frac{d^{2}}{d u^{2}}+\frac{1}{u} \frac{d T}{d u}-T=0
$$

The theory of Bessel functions it is proved that the solution of the equation is:

$$
T=C_{1} I_{0}(u)+C_{2} K_{0}(u)
$$

Sizes $I(u)$ and $K(u)$ it is possible to find in special tables [13-15].

Any constants of $S_{i I}{ }^{2}$ can be received from boundary conditions.

Conditions of symmetry follow that in the center of a grate derivative of temperature on coordinate it is equal to zero. Then on the basis of the equation (22) it is possible to write:

$$
\frac{d T}{d u} C_{1} I_{1}(0)+C_{2} k_{1}(0)
$$

That temperature in the center of a grate had final value, coefficient2 With at $k(0)$ it has to be equal to zero. The coefficient $\mathrm{C} 1$ be defined, knowing that at $u=u_{a}, T=T_{l}$. Finally we receive:

$$
T=T_{1} \frac{I_{0}(u)}{I_{0}\left(u_{a}\right)}=\frac{I_{0}\left(\sqrt{\frac{k}{\sigma}} \frac{x}{\sqrt[3]{h}}\right)}{I_{0}\left(\sqrt{\frac{k}{\sigma}} \frac{a}{\sqrt{h}}\right)}
$$

Let's pass to numerical calculations. It is known that at a temperature $1000{ }^{\circ} \mathrm{C}$ each square centimeter of a steel product depending on air circulation around it and a condition of a surface can lose from 10 to $30 \mathrm{~W}$. Therefore coefficient $k$ for Newton's law of a thermolysis it is necessary to take from 0.01 to $0.03 \mathrm{~W} / \mathrm{cm} 2 \cdot{ }^{\circ} \mathrm{C}$. Heat conductivity became, we will accept $0.25 \mathrm{~W} / \mathrm{cm} 2 \cdot{ }^{\circ} \mathrm{C}$.

Consequently, the ratio of the heat transfer coefficient to the thermal conductivity $\mathrm{k} / \sigma$ will be in the range of $0.04-0.12 \mathrm{~cm}^{-1}$, and the square root of this ratio is $0.2-0.35 \mathrm{~cm}^{-1 / 2}$. The temperature at the center of the grate will be related to the temperature at the edge of the disk by the equation

$$
T_{1}=T_{0} I_{0}\left[(0,2-0,35) \frac{a}{\sqrt{h}}\right]
$$

For practical calculations Bessel's function is allowed to be replaced with an exponent. We will postpone the beginning of coordinates to a working edge of a grate, then

$$
T=T_{1} \exp \left[-\sqrt{\frac{k}{\sigma}} \frac{x}{\sqrt{h}}\right]
$$

At a temperature of hardening of $850^{\circ} \mathrm{C}$ we will take overheating $100{ }^{\circ} \mathrm{C}$. The relation of $\mathrm{T}$ and $\mathrm{T} 1 \mathrm{will}$ be, therefore, 0,9 . For observance of this condition the argument exhibitors has to be no more than 0.1 , therefore, thickness of the tempered layer $\frac{x^{2}}{h}=0,2 \mathrm{ci}$.

Let's take a case when $z_{a} \mu \ll a$, we will also consider, voltage drop on the inductor almost does not depend on its ohmic resistance and is defined only by its inductance [12]: 


$$
E_{i}=I_{i} L_{i} \omega
$$

Let's consider that the stream of vortex current in a grate has the same width of $g$, as a tape of the inductor, and length of the average line of vortex current is approximately equal to inductor length $l_{i}$. Then the power multiplier (ohmic resistance of a current-carrying layer of a grate) will be equal:

$$
R_{a}=\frac{\rho_{a} l_{i}}{g z_{a}}
$$

As the heated grate surface, that equation (28) can be rewritten:

$$
R_{a}=\frac{\rho_{a} l_{i}^{2}}{S_{a} z_{a}}
$$

On the basis of (27) and (28) it is possible to write that the power allocated in a product is equal:

$$
P_{a}=I_{i}^{2} R_{a}=\frac{E_{i}^{2} l_{i}^{2} \rho_{a}}{L_{i}^{2} \omega^{2} S_{a} z_{a}}
$$

Having substituted instead of penetration depth and having made necessary reductions in the equation (30), we will receive:

$$
P_{a}=\frac{E_{i}^{2} l_{i}^{2}}{L_{i}^{2} S_{a} 2 \pi}\left(\rho_{a} \mu_{a}\right)^{\frac{1}{2}} f^{-\frac{3}{2}}
$$

If when hardening the distance between a grate and the inductor is not enough in comparison with width of the conductor of inductor $\mathrm{g}$, then the inductance of a system a grate inductor will be equal:

$$
L_{i}=\frac{4 \pi a l_{i}}{g}
$$

Let's divide both members of equation (31) into the heated area. Then on the left side of the equation there will be not a full capacity.

The equation (31) can be presented in the form now

$$
\Delta P=\frac{E_{i}^{2} l_{i}^{2}}{\left(\frac{4 \pi a l_{i}}{g}\right)^{2} l_{i}^{2} g^{2} 2 \pi}\left(\rho_{a} \mu_{a}\right)^{\frac{1}{2}} f^{-\frac{3}{2}}
$$

After necessary reductions we will receive:

$$
\Delta P=\frac{E_{i}^{2}}{32 \pi^{2}\left(a l_{i}\right)^{2}}\left(\rho_{a} \mu_{a}\right)^{\frac{1}{2}-\frac{3}{2}}
$$


This formula gives mutual communication between specific power, current frequency, and tension on the inductor, physical characteristics of the heated metal geometrical sizes of the inductor. Inductor g width is not included into this formula.

When performing practical calculations on the basis of a formula (34) one of the sizes entering it is taken for required function, the second - for an independent variable, and all other sizes - for parameters.

When calculating we will take at first for function frequency, and for an independent variable - the inductor sizes; we will consider parameter tension square relation on the inductor specific power. Then in practical units the formula (34) will be written:

$$
f=10^{7}\left(\frac{E_{i}^{2}}{\Delta \mathrm{P}}\right)^{\frac{2}{3}}\left(\rho_{a} \mu_{a}\right)^{\frac{1}{3}}\left(l_{i} a\right)^{-\frac{4}{3}}
$$

the first case (modification steel $\alpha$ ) the formula (35) takes a form:

$$
f=10^{6}\left(\frac{E_{i}^{2}}{\Delta \mathrm{P}}\right)^{\frac{2}{3}}\left(l_{i} a\right)^{-\frac{4}{3}}
$$

For steel in an austenitic state (modification steel) the formula has an appearance:

$$
f=4,8 \cdot 10^{5}\left(\frac{E_{i}^{2}}{\Delta \mathrm{P}}\right)^{\frac{2}{3}}\left(l_{i} a\right)^{-\frac{4}{3}}
$$

In case of use of the cylindrical inductor diameter length of its conductor, in this case the formula (35) can be written:

$$
f=2,1 \cdot 10^{6}\left(\frac{E_{i}^{2}}{\Delta \mathrm{P}}\right)^{\frac{2}{3}}\left(d_{i} a n\right)^{-\frac{4}{3}}
$$

Formulas (35) and (37) show that with increase in length of the inductor it is necessary for maintaining former values $\mathrm{E}$ to lower the frequency of the feeding current. With the set frequency and specific power tension on the inductor grows in proportion.

For determination of tension on the inductor it is necessary that it was taken as function. An independent variable in this case - specific power at the same time it is accepted, and parameter - current frequency. Then the equation looks so:

$$
E_{i}^{\prime}=1,8 \cdot 10^{-5} f^{\frac{3}{4}}\left(\rho_{a} \mu_{a}\right)^{-\frac{1}{4}} \Delta \mathrm{P}^{\frac{1}{2}}
$$

As the above theoretical studies show, high-frequency heat treatment differs quite significantly in speed and nature of cooling from conventional heat treatment with heating in furnaces. These differences are especially noticeable during surface and local heating, when the surface of the cooled metal is part of the volume of the entire product.

If to heat superficially small site of a big and thick steel detail, then its cooling even on air occurs very quickly.

As an example it is possible to consider heating and hardening of a round body - in 
bulk. If depth of the layer heated to temperature of hardening, making $10 \%$ of size of shaft radius (for example, $2 \mathrm{~mm}$ with a diameter of $40 \mathrm{~mm}$ ) and a transitional layer which temperature of heating falls from hardening to initial also $10 \%$, then total amount of the entered heat is enough for heating of all shaft to temperature which is $30 \%$ of hardening. For example, $250^{\circ} \mathrm{C}$ at a temperature of hardening of $850{ }^{\circ} \mathrm{C}$, with a depth of layer of $20 \%$ from radius and existence of the same transitional layer the average temperature of a shaft after alignment reaches it about $50 \%$ of temperature of hardening and will be equal $425{ }^{\circ} \mathrm{C}$ at a temperature of hardening of $850{ }^{\circ} \mathrm{C}$.

The warming up of a core leads to sharp delay of cooling at temperatures above a martensitic point and can interfere with receiving martensite.

At superficial hardening of the working area of a grate (up to the depth of $2 \mathrm{~mm}$ ) at a thickness of $17 \mathrm{~mm}$ there can be a core warming up at small speeds of heating. Use of cooling liquid will sharply accelerate cooling process.

Cooling of a grate with immersion in liquid (in water) is not expedient since so far surface temperature considerably (by hundreds of degrees) exceeds liquid boiling temperature, on the cooled surface steam(period of film boiling) is created and keeps. This film reduces intensity of process of cooling.

The most convenient way of cooling when hardening grates is cooling with a water shower.

At simultaneous hardening of souls allows to make cooling on the place without transfer of a grate in a hardening tank owing to what cooling can be begun through fractions of a second after the end of heating. At consecutive hardening the application of a shower creates still big advantages as at such hardening the cooling with immersion does not provide uniformity of cooling that results in danger of formation of cracks and to emergence of soft spots.

On the basis of the above-stated mathematical models it is calculated and it is recognized as optimum, for the grate made of brand 45 steel hardening temperature at a surface $960{ }^{\circ} \mathrm{C}$ and on border of a heated layer $850{ }^{\circ} \mathrm{C}$.

\section{Results}

At high-frequency superficial hardening the large role is played by the hardening mode. For providing the mode of hardening, first of all, current. It is chosen according to the sizes of a grate and the chosen depth of the tempered layer - $8000 \mathrm{~Hz}$.

Based on the selected parameters, an inductor with a water shower was designed, design documentation was developed, a prototype was manufactured and mounted on an highfrequency current generator.

For the purpose of determination of quality of hardening of a working surface of grates, researches on determination of hardness and wear resistance were conducted. Hardness was measured on two sites - on tempered and on not tempered, for comparison purposes.

As showed results of tests, hardening of a surface of the working area reaches surface hardness to HRC 55 that allows, to increase the term of operation of grates by more than two times.

Comparative wear steel after hardening and pig-iron grates are investigated on special bench installation [2]. Results of researches are given in the table.

Apparently from data the provided in the table, steel tempered grates had smaller wear for an identical period due to hardening of a side surface.

At the next stage of this work, industrial tests of hardened grates were carried out. On two gins of an experimental cotton factory, five hardened and not hardened steel grates were installed in a grate made of cast iron grates. 
Table 1. Research results of comparative wear of hardened steel and serial cast iron grates

\begin{tabular}{|c|c|c|c|c|}
\hline \multirow{2}{*}{$\begin{array}{c}\text { Time, } \\
\text { min }\end{array}$} & \multicolumn{2}{|c|}{ The tempered steel grate } & \multicolumn{2}{c|}{ Serial cast iron grate } \\
\cline { 2 - 5 } & Wear, micron & $\begin{array}{c}\text { Area of contact, } \\
\mathbf{m m}^{\mathbf{2}}\end{array}$ & Wear, micron & $\begin{array}{c}\text { Area of contact, } \\
\mathbf{m m}^{\mathbf{2}}\end{array}$ \\
\hline 4 & 55 & 29 & 104 & 35 \\
\hline 6 & 80 & 33 & 100 & 40 \\
\hline 12 & 115 & 66 & 130 & 72 \\
\hline 30 & 210 & 84 & 250 & 93 \\
\hline 60 & 270 & 132 & 310 & 140 \\
\hline 90 & 350 & 165 & 380 & 174 \\
\hline 120 & 380 & 190 & 450 & 194 \\
\hline
\end{tabular}

As a result of observations within three months it is established that operation term at the tempered grates is more than 2 times higher, than at not tempered. Such indicators allow using one set of grates within one season during capital repairs, unlike use of two sets having now. On the basis of these researches it is recommended to use the tempered grates in the industry.

\section{Conclusions}

1) Change of a gap between grates in a grate lattice leads to change of parameters of process of ginning and finally to deterioration of products.

2) Mathematical models for calculations of parameters of process of hardening of the working area of grates are received.

3) On the received mathematical models key parameters of process of hardening of high-frequency current which have the following values are calculated:

a. depth of the tempered layer $-2.0 \mathrm{~mm}$.

b. optimum frequency of current $-60000 \mathrm{~Hz}$.

c. specific power $-500 \mathrm{~W} / \mathrm{cm}^{2}$.

d. hardening time - $10 \mathrm{sec}$.

4) Studying hardness tempered and not tempered sites of grates by method of Rockwell showed increase in hardness of the tempered site to HRC 55 against HRC 45 at not tempered.

5) Production tests of gin grates with a hardened working zone "Khlopkoprom" showed an increase in the stability of the ginning process while maintaining the quality indicators of products and confirmed the results of theoretical and bench studies.

\section{References}

1. A. Ismailov, R. Makhkamov, A. Ibragimov, M. Agzamov, J. Problems of textiles 3, 10 (2005)

2. M. Agzamov, S. Yunusov, J. Gafurov, IOP Conference Series: Materials Science and Engineering 254(8), 082017 (2017)

3. D. Muhammadiev, Kh. Akhmedov, B. Primov, F. Ibragimov, L. Zhamolova, T. Muhammadiev, Izvestiya Vysshikh Uchebnykh Zavedenii, Seriya Teknologiya Tekstil'noi Promyshlennosti 2020(2), 88-92 (2020)

4. R. Rosulov, Izvestiya Vysshikh Uchebnykh Zavedenii, Seriya Teknologiya Tekstil'noi Promyshlennosti 2017(1), 119-122 (2017)

5. P. Bernard, Textiles fiber to fabric, 593 (McGraw-Hill International editions Press, New York, 1988) 
6. M. Agzamov, Izvestiya Vysshikh Uchebnykh Zavedenii, Seriya Teknologiya Tekstil'noi Promyshlennosti 2007(1), 26-29 (2007)

7. M. Agzamov, M. Agzamov, G. Madgidov, Izvestiya Vysshikh Uchebnykh Zavedenii, Seriya Teknologiya Tekstil'noi Promyshlennosti 2007(3), 34-37 (2007)

8. R. Makhkamov, M. Agzamov, Izvestiya Vysshikh Uchebnykh Zavedenii 2006(2), 101104 (2006)

9. S. Yunusov, European applied sciences 3, 79-85 (2015)

10. G. Holt, W. Laird, The Journal of Cotton Science 11, 79-90 (2017).

11. M. Agzamov, A. Kh. Inagamov, M. M. Agzamov, American Journal of Mechanical and Materials Engineering 33-39 (2019)

12. S. A. Ismail, G. Chen, C. Baillie, T. Symes, Biosystems Engineering 109(2), 140-147 (2011) 